\title{
A study of the effect of saliva on the concentration of mucin in gastric juice and its possible relationship to the aetiology of peptic ulcer
}

\author{
S. L. MALHOTRA
}

From the Medical Department, Western Railway, Bombay, India

EDITORIAL COMMENT This interesting paper suggests that the consistency of food affects the quality of gastric mucus derived from swallowed saliva, foods needing much mastication stimulating a high salivary output. Salivary mucus, by augmenting gastric mucus, may help to protect against peptic ulceration.

The two most important protective barriers against peptic ulceration are the surface epithelial cells themselves with their astounding capacity for regeneration and the film of mucus which covers them. The remarkable regenerative capacity of the epithelial cells has been studied by various workers with identical conclusions (Grant, 1945; Palmer, 1952; Grant, Grossman, and Ivy, 1953), from which it is evident that erosion and repair are repeatedly taking place as a result of the repeated trauma of digestion, but the process of regeneration is carried on with such facility that this trauma does not have the inflammatory results which it might have elsewhere.

Much work has also been done which shows that the effects of mechanical irritation of the gastric mucosa as well as of eating are similar in that these result in an increased outpouring of mucus from the epithelium (Wolf and Wolff, 1948).

It was shown in an earlier report that differences in the pattern of diet and eating produced variations in the total quantities, the buffer capacity, and the mucin content of saliva (Malhotra, 1965; Malhotra, Saigal, and Mody, 1965), and from these studies it was obvious that, apart from influencing gastric acidity (Malhotra, 1967a), swallowing of saliva will also influence, to a variable extent, the total amount of mucus present in the gastric lumen, because not only the total quantities of saliva swallowed with food, but also the concentration of mucus in the saliva, vary with the pattern of diet and eating (Brown, Wright, and Limbacher, 1938; Dewar, 1952; Dewar and Parfitt, 1954; Malhotra, 1965; Malhotra et al., 1965). This paper attempts to study by a quantitative method the effect of saliva on mucus in the gastric juice.

\section{METHOD AND THE DESIGN OF EXPERIMENTS}

In designing the experiments an attempt has been made to 'exclude' saliva from the stomach in one series of observations and allow its admixture with food, to the extent that it is normal during eating, in the other. This was accomplished by giving the meal by mouth in one set of experiments and through an intranasal Ryle's tube, positioned in the fundus of the stomach fluoroscopically, in the other. In the experiments in which it was desired to 'exclude' saliva the subjects were instructed not to swallow saliva but to eliminate it by frequent spitting, whilst in the experiments designed to study the effect of saliva, they swallowed it with the food, as it is normally done.

THE MEALS Both solid and fluid meals were used. A description of the solid 'sloppy' and 'masticatory' meals has been given elsewhere (Malhotra et al., 1965). The liquid meal was the South Indian appetiser called rasam. The $p \mathrm{H}$ of the various batches of rasam ranged between 4.3 and $5 \cdot 2$, the majority being of $p \mathrm{H} 4 \cdot 6$. To eliminate any possible bias due to $p \mathrm{H}$ variations, the same pool of the liquid meal was used for 'oral' and the 'tube' experiment.

THE SUBJECTs Thirty-two adult males, who included ambulant patients from the wards of the Jagjivan Ram Hospital, free from gastric or small bowel disease, and healthy volunteers, picked randomly from among the class IV staff of the Hospital, and ranging in age from 17 to 53 years, were included in experiments by random allocation into four groups as follows:

Group $A$ This comprised 13 persons: to seven, boiling hot rasam was given sip by sip in small sips orally, to encourage admixture with saliva in four runs (a to d) of $100 \mathrm{ml}$. each and subsequently at room temperature through the Ryle's tube in four runs (e to $h$ ) so that saliva 
was excluded; in another six the procedure was reversed to eliminate the effect if any, due to changes in stimulus intensity, viz., the first four runs were given through the tube and the last four runs orally.

Group $B$ This comprised seven persons: to three, rasam at room temperature was given through the tube (a to d) and then orally as a 'long drink' (e to h) as it is normally taken in South India; in the remaining four the order was reversed.

Group $C$ This comprised six persons in whom the 'masticatory' solid meal was used: in three orally on the first day and on the next day through the Ryle's tube made into a paste. In the other three the order was reversed.

Group D Of six persons, the South Indian style sloppy meal of boiled rice and lentil soup was used in the same manner as the Punjabi masticatory food.

GASTRIC JUICE SAMPLES To obtain samples, the method of Brooks, Erskine, Gephart, Swaim, and Moore (1950) was used for groups $A$ and $B$.

In this, the stomach is completely emptied of its fasting contents and $100 \mathrm{ml}$. of the fluid meal marked with a dye (to determine the secretory and emptying rates), as described elsewhere (Malhotra, 1967b), is given orally or through the tube as the case may be. After allowing the meal to 'digest' for 10 minutes, the residue was recovered. Eight such successive runs were carried out and labelled alphabetically a to $h$ and all specimens analysed. Specimens $a$ and $b$ and $e$ and $f$ have not been taken into account, as basal conditions do not prevail until after the first two runs (Brooks et al., 1950) and, moreover, these help to wash out any residual mucin left over from the fasting contents.

In the case of the solid meals, because of the difficulty of complete aspiration, the experiments were done on two different days. Two equal and identical portions of the meals were used, one of which was eaten in the normal manner on one day so that saliva mixed with the meal and the other was made into a thin paste and was introduced through the tube, on the succeeding day, so that the saliva was excluded from the meal. Two hundred $\mathrm{ml}$. of normal saline was allowed for each portion, in one case to make the paste from the meal, and in the other the subject swallowed this by mouth, at the end of the meal. With this mode of investigation, each patient supplied eight samples (excluding the fasting contents): four with saliva and four with no saliva. When working with 'sloppy' meals (group D) we overcame the difficulty of residual salivary mucus by preceding the experiments with two saline runs of $100 \mathrm{ml}$., each which served as a washout. Unfortunately, due to an initial error in planning this step was not taken in the case of the solid 'masticatory' meals (group C).

\section{ESTIMATION OF MUCIN}

The Congo red and acid-alcohol (A.C.R.A.) method of Burnet (1948), which is based on the fact that if a mucus solution is dropped into acid-alcohol a firm pellicle forms at the interface, maintaining the drop either in globular or with weaker solution in annular form, has been used previously for the quantitation of salivary mucus (Dewar and Parfitt, 1954; Malhotra et al., 1965). The shapes can be rendered easily visible by adding an alcohol-insoluble dye to the mucus solution. In the absence of mucus the drop flattens out immediately and leaves only a cloudy patch without a definite margin. The method is very quick and results are reproducible within the required order of accuracy (Burnet, 1948).

For the present study, the A.C.R.A. method of mucus estimation has been adapted to gastric juice. If a solution containing mucus and saliva is allowed to stand at room temperature, $30 \%$ of mucus is destroyed within 30 minutes and $50 \%$ in two hours. But if the sample is placed on ice immediately after collection, destruction is only slight (Dewar and Parfitt, 1954; Malhotra et al., 1965). In order to obtain comparable results we had, therefore, collected our samples in stoppered glass cylinders surrounded with ice. Sampling was done in a room adjoining the work bench to facilitate immediate assay. On the completion of full gastric aspiration, the glass cylinders were vigorously shaken to homogenize the mucus and $0.5 \mathrm{ml}$. from this was immediately (within two minutes) transferred to a test tube for serial two-fold dilutions with saline buffered with borate at $p \mathrm{H} 7 \cdot 1$. To each tube 2 drops of $0 \cdot 1 \%$ Congo red solution in water were added. In cases where the first tube was highly acidic and the indicator turned blue, one drop of $1 \mathrm{~N} \mathrm{NaOH}$ was added. This step not only clarified the end point but also helped to homogenize the mucus by dissolving it. While making the serial dilutions homogenization and thorough mixing was secured in the tubes by rapid up-and-down movement of the solution in a teat pipette with a wide opening.

To record the results the original procedure of Burnet (1948) was used, in which one drop from each tube is dropped gently into a shallow layer of acid-alcohol (about $4 \mathrm{~mm}$.) in a flat petri dish with transparent bottom. The acid-alcohol is a $1 \%$ solution of strong $\mathrm{HCl}$ in $70 \%$ alcohol. The results are recorded as A.C.R.A. titres, employing identical end-points as in Burnet's original technique of mucin assay. The great advantage of the A.C.R.A. method is the simplicity with which it can be performed to yield reproducible results, and its sensitivity, as it can measure even very minute amounts of mucus in solution.

The tests were done immediately on collection, using a 'blind' technique in which the observer noting the end points was not aware of the origin of the samples. Serial dilutions of every batch of the fluid meals, and, in the case of the solid meals, similar dilutions of $20 \%$ aqueous extract of the meal paste were tested for the presence of mucus. None of these contained mucus.

\section{RESULTS}

The results of the mucin content of the samples of gastric juice have been expressed as A.C.R.A. titres. For the purpose of the analysis of the results, the titres have been grouped in ranges as shown in Table I. 
TABLE I

DISTRIBUTION OF SAMPLES OF GASTRIC CONTENTS IN DIFFERENT A.C.R.A. TITRE RANGES OF SALIVARY MUCUS

Siudy Sample A.C.R.A. Titres ${ }^{1}$

No. of Samples No. of Samples Showing Positive A.C.R.A. Titres in Different Tube Ranges of Samples

Showing Negative

A.C.R.A. Titre

in First Tube

\begin{tabular}{|c|c|c|c|c|c|c|c|c|}
\hline & & 0 & 1-3 & 4-6 & $7-9$ & $10-12$ & 13 & \\
\hline \multirow[t]{2}{*}{$\mathbf{A}$} & With saliva & 0 & 9 & 6 & 6 & 4 & 1 & 26 \\
\hline & With no saliva & 18 & 6 & 0 & 2 & 0 & 0 & 26 \\
\hline \multirow[t]{2}{*}{ B } & With saliva & 2 & 8 & 3 & 1 & 0 & 0 & 14 \\
\hline & With no saliva & 7 & 6 & 1 & 0 & 0 & 0 & 14 \\
\hline \multirow[t]{2}{*}{ C } & With saliva & 0 & 10 & 3 & 8 & 3 & 0 & 24 \\
\hline & With no saliva & 9 & 7 & 5 & 2 & 1 & 0 & 24 \\
\hline \multirow[t]{2}{*}{ D } & With saliva & 13 & 3 & 5 & 2 & 1 & 0 & 24 \\
\hline & With no saliva & 15 & 3 & 4 & 2 & 0 & 0 & 24 \\
\hline
\end{tabular}

Study A: fluid meal

Comparison in 13 individuals, between sip by sip ingestion versus introduction of meal through Ryle's tube.

Study B: fuid meal

Comparison in seven individuals, between ingestion as a 'long drink' versus introduction of meal through Ryle's tube.

Study C: solid 'masticatory' meal

Comparison in six individuals, between ingestion by thorough mastication versus introduction of meal as a paste through Ryle's tube.

Study D: solid 'sloppy' meal

Comparison of six individuals, between ingestion by bolting down versus introduction of meal as a paste through Ryle's tube.

In studies A and B with fluid meals each person supplied four samples, two with saliva and two with no saliva.

In studies $\mathbf{C}$ and $\mathbf{D}$ with solid meals each person supplied eight samples, four with saliva and four with no saliva.

${ }^{1}$ The figures indicate tube numbers for dilution, e.g., 1 st $=1: 1 ; 2$ nd $=1: 2 ; 3$ rd $=1: 4 ; 4$ th $=1: 8$ and so on.

COMPARISON OF A.C.R.A. TITRES OF GASTRIC CONTENTS AFTER FLUID MEALS AND MEALS THROUGH RYLE'S TUBE Mucin in high titres was invariably present in the fasting samples ranging from a titre of $1: 1$ to $1: 256$ (first to ninth tube).

Mucin was completely absent in 18 samples out of $26(69 \%)$ when saliva was 'excluded' from the meals, in contrast to nil out of 26 samples with saliva. There were more samples with high A.C.R.A. titres going up to the thirteenth tube $(1: 4,096)$ when saliva was admixed with the meal, as compared with when it was excluded.

COMPARISON OF A.C.R.A. TITRES OF GASTRIC CONTENTS AFTER FLUID MEALS AS A LONG DRINK VERSUS THROUGH THE RYLE'S TUBE All fasting samples showed a high concentration of A.C.R.A. titre ranging from $1: 2$ to 1:256 (second to ninth tube).

Mucin was completely absent in seven out of 14 samples $(50 \%)$, when the meals were given through the Ryle's tube thus 'excluding' saliva. The majority of the samples with saliva $(90 \%)$ were distributed between the lower titres of $1: 1$ to $1: 8$ (first to fourth tube). When these results are compared with the results of sample obtained in study $A$, in which the fluid meals were taken sip by sip, to encourage admixture with saliva, it was seen that the concentration of mucin in the aspirates was much higher with the latter mode, namely, study $\mathbf{A}$, as compared with the former, namely, study B (Table I).

COMPARISON OF A.C.R.A. TITRES OF GASTRIC CONTENTS AFTER MASTICATORY SOLID MEALS BY MOUTH VERSUS
A PASTE INJECTED THROUGH RYLE'S TUBE With solid masticatory meals mucin was present in all samples whether taken by mouth or through the tube but the A.C.R.A. titres were higher in samples obtained during experiments when saliva was swallowed than when it was 'excluded'. We had made an initial mistake in our planning that with the solid 'masticatory' meals we did not wash out the stomach with saline to bring about 'basal' conditions and, therefore, some mucin may have been left in the stomach from the residual fasting juice despite our attempts to empty it out with the fasting contents. One possibility, therefore, arises that because of incomplete removal of mucus from the fasting contents, the presence of mucin in samples obtained when saliva was 'excluded' may really have been due to the presence, in the gastric lumen, of residual salivary mucus ordinarily present in the fasting contents, due to the swallowing of saliva during sleep. Many particles of mucus adhere to the gastric wall and are not recovered through the tube. This is the general opinion of gastroscopists and it has been observed directly through a gastric fistula by Wolf and Wolff (1948). This possibility is confirmed from our experiments with 'sloppy' meals in which a saline washout preceded the collection of samples and also when we repeated the procedures with masticatory meals on two other patients, not included in this series, with a saline washout to precede the experiments.

COMPARISON OF A.C.R.A. TITRES OF GASTRIC CONTENTS WITH SLOPPY SOLID MEALS BY MOUTH VERSUS AS A PASTE INJECTED THROUGH RYLE'S TUBE The samples 
with saliva and with no saliva were equally distributed between different A.C.R.A. ranges. There were more samples showing a complete absence of mucus in this mode of investigation in contrast to the masticatory meals (study $\mathrm{C}$ ). This can be explained by the fact that larger amounts of saliva are swallowed with masticatory meals as compared with sloppy food (Dewar and Parfitt, 1954; Malhotra et al., 1965).

ADEQUACY OF THE METHOD The A.C.R.A. method of quantitating mucus has been previously used for the salivary mucus and has been found to give reproducible results within the required order of accuracy (Dewar, 1952; Dewar and Parfitt, 1954; Knox and Still, 1953; Malhotra et al., 1965). It has not been previously used for quantitating the gastric mucus, and the question whether this method determines only the salivary mucus and ignores the gastric mucus must therefore be carefully considered. To investigate this possibility, we introduced a test meal of $100 \mathrm{ml}$. of $5 \% \mathrm{NaHCO}_{3}$ in distilled water into the stomach through the Ryle's tube on the completion of the main experiment in six of our patients who had shown no mucus in samples obtained after the tube feeding. Ten minutes were allowed for this meal to 'digest'. Although the A.C.R.A. titre in samples preceding the $\mathrm{NaHCO}_{3}$ instillation were negative in all these patients, without exception, high A.C.R.A. titres were found after the alkali meal (in one case up to the twelfth tube). Since saliva had been carefully excluded from these samples, this clearly shows that the high A.C.R.A. titres in these samples must be due to mucus of gastric origin, because with a fall in acidity the intracellular mucus from the gastric epithelium is removed and goes into solution. Our results cannot, therefore, be due to any inadequacy of the method to measure the concentration of gastric mucus, and obviously the A.C.R.A. method of quantitating mucus is applicable to both the salivary as well as gastric components of mucus.

Is it possible that if we have not succeeded in aspirating the gastric mucus through the Ryle's tube it is because mucus precipitates into thick strands on coming into contact with acid and may have thus offered resistance to aspiration? This appears unlikely as it should have equally affected the salivary mucus swallowed with the meals, which, on the other hand, we were able to aspirate without difficulty. Moreover, in the studies of James and Pickering (1949), as in ours, using aspiration after consumption of solid food, it was sometimes surprising how thick the material was which came up the Ryle's tube, the sample occasionally being of a consistency such that it could hardly be poured.

A final source of error can be introduced in the method if the mixing of the mucus in the gastric juice by thorough shaking of the cylinder is inadequate. Glass and Boyd (1948) have shown that if gastric juice containing saliva is centrifuged part of the salivary mucus collects on the bottom and at the top of the centrifuge tube. We employed vigorous shaking in a glass stoppered jar to secure homogenization of the mucus, so producing homogeneous dispersion of the salivary and gastric mucus components in the fluid. This is shown by the following test:

Test for mixing In six of our samples (from different individuals), after the assay of the mucus in the usual manner, we added $1 \mathrm{~N} \mathrm{NaOH}$ to bring the $p \mathrm{H}$ to 9 and repeated the assay. The samples without saliva showed no mucus by both the methods. The samples with saliva showed similar results (within \pm 1 dilution) with both the methods. Since in an alkaline medium mucus goes into solution, it is clear that our method of vigorous shaking had secured nearly complete mixing and homogenization of both the mucus components, as otherwise the results from this test could not have been comparable with the results of the main experiment.

The inference of these results, therefore, is that (1) larger amounts of mucus are present in the stomach when saliva is added as compared to when it is not; (2) mucus in gastric aspirates obtained after serial test meals is chiefly of salivary origin and hardly any from the gastric mucous membrane, because in the samples with no saliva mucus was generally absent or in very low concentrations.

EFFECT OF THE CEPHALIC PHASE OF GASTRIC SECRETION ON GASTRIC MUCUS A legitimate criticism of our method in which we gave the meal by mouth in one set of experiments and by intubation in the other, is that the differences in mucus concentration noted by us between the samples with saliva versus the samples with no saliva may really be due to the possibility that important secretory events associated with the cephalic phase of gastric secretion may be lacking in the situation in which food was passed directly through the tube into the stomach. This has been tested in the following manner:

Seventeen convalescent patients admitted to the Jagjivan Ram Hospital for diseases unrelated to the gastrointestinal tract were included in the test. From a common pool of the fluid meal, for each patient we secured admixture of saliva with the meal in four aliquots by asking the patients to sip the meal, keep each sip in the mouth in order to encourage admixture with saliva, and then collect it in a marked receptacle. To pair with these aliquots, equal volumes of the meal from the same pools were taken. Thus we had four pairs of test meals for each 
patient, namely, four test meals with saliva and four test meals with no saliva, which were in this test administered by intubation after the evacuation of fasting contents and using two runs each of $100 \mathrm{ml}$. of distilled water as a stomach wash. The experiments in this test too were done in a room adjoining the work bench and the patients were instructed not to swallow saliva but to eliminate it by spitting, this being carefully supervised. Thus the possibility of important secretory events associated with the cephalic phase of gastric secretion and a slower rate of gastric filling affecting the results was eliminated in this test, since all the test meals were given by intubation. On the completion of the runs of test meals, we intubated $100 \mathrm{ml}$. of $5 \% \mathrm{NaHCO}_{3}$, allowed it to 'digest' for 10 minutes, and completely withdrew the contents for similar analysis.

The results of the test in Table II show that in 14 out of 17 patients the samples with no saliva

\section{TABLE II}

RESULTS OF TEST TO SEPARATE EFFECT OF REPEATED SWALLOWING, TEMPERATURE OF THE MEAL, AND THE CEPHALIC PHASE OF GASTRIC SECRETION

\begin{tabular}{|c|c|c|c|c|c|c|c|c|c|c|c|}
\hline \multirow{3}{*}{$\begin{array}{l}\text { Patient } \\
\text { No. }\end{array}$} & \multirow[t]{3}{*}{ Age } & \multicolumn{10}{|c|}{ A.C.R.A. Titre } \\
\hline & & \multicolumn{5}{|c|}{ Sample with Saliva } & \multicolumn{4}{|c|}{$\begin{array}{l}\text { Sample with no } \\
\text { Saliva }\end{array}$} & \multirow[b]{2}{*}{$A$} \\
\hline & & $F$ & $a$ & $b$ & $c$ & $d$ & $e$ & $f$ & $g$ & $h$ & \\
\hline 1 & 41 & 6 & 4 & 4 & 3 & 4 & 0 & 0 & 0 & 0 & 4 \\
\hline 2 & 25 & 2 & 1 & 8 & 1 & 1 & 0 & 0 & 0 & 0 & 4 \\
\hline 3 & 30 & 8 & 3 & 1 & 3 & 3 & 0 & 0 & $\mathbf{0}$ & 0 & Not done \\
\hline 4 & 31 & 3 & 5 & 7 & 4 & 4 & 0 & 0 & 0 & 0 & 3 \\
\hline 5 & 35 & 3 & 5 & 4 & 0 & 1 & 0 & 0 & 0 & 0 & 3 \\
\hline 6 & 18 & 4 & 2 & 3 & 2 & 3 & 2 & 9 & 3 & 1 & 7 \\
\hline 7 & 20 & 5 & 3 & 1 & 1 & 1 & 1 & 1 & 0 & 0 & 5 \\
\hline 8 & 38 & 1 & 2 & 3 & 3 & 2 & 1 & 0 & 0 & 0 & 2 \\
\hline 9 & 21 & 7 & 5 & 1 & 1 & 1 & 0 & 0 & 0 & 0 & 3 \\
\hline 10 & 50 & 5 & 2 & 1 & 3 & 2 & 0 & 0 & 0 & 0 & 3 \\
\hline 11 & 27 & 5 & 6 & 2 & 7 & 7 & 0 & 0 & 0 & 0 & 5 \\
\hline 12 & 31 & 2 & 3 & 1 & 5 & 1 & 0 & 0 & 0 & 0 & 2 \\
\hline 13 & 48 & 5 & 4 & 4 & 4 & 8 & 2 & 8 & 9 & 8 & 8 \\
\hline 14 & 23 & 4 & 9 & 2 & 5 & 1 & 0 & 0 & 0 & 0 & Not done \\
\hline 15 & 25 & 5 & 2 & 2 & 7 & 5 & 0 & 0 & 0 & 0 & 1 \\
\hline 16 & 51 & 5 & 1 & 0 & 0 & 3 & 2 & 3 & 2 & 0 & 2 \\
\hline 17 & 34 & 3 & 1 & 5 & 5 & 1 & 0 & 0 & 0 & 0 & 7 \\
\hline
\end{tabular}

Comparison of the A.C.R.A. titres between samples with saliva and samples with no saliva in 17 individuals, the test meals being administered by intubation. The figures represent the tube numbers.

$F=$ fasting, a to $h$ are the different runs of test meals: in patients 1 to 9 the runs with saliva preceded the runs with no saliva whereas in patients 10 to 17 this was reversed. $A=$ the run in which the test meal was $100 \mathrm{ml}$. of $5 \% \mathrm{NaHCO}_{3}$. The figures of the A.C.R.A. titre stand for the tube numbers, showing the end point.

were negative for mucus whereas the samples with saliva showed the presence of mucus in varying concentrations. This shows that the differences we noted are not due to the cephalic phase of gastric secretion or to its effect on the secretion of mucus by the gastric mucus membrane. The fasting contents as well as the samples in which the test meal was $5 \% \mathrm{NaHCO}_{3}$ were invariably positive. Cases 6,13 , and 16 showed high A.C.R.A. titres in the samples with saliva as well as in the samples with no saliva. All of these patients had a marked duodenal reflux with heavy bile contamination of the samples, which may explain the high A.C.R.A. titres in their samples with no saliva. Case 13 gave a history of chronic gastritis and a healed gastric ulcer.

\section{DISCUSSION}

The results of our studies with fluid as well as solid meals show unequivocally that mucus was either completely absent or present in extremely low concentrations in samples from which saliva had been 'excluded'. In contrast to this, mucus in varying concentration was invariably present in samples withdrawn after eating meals with which saliva was allowed to mix as well as in the fasting samples. Since the various foods employed as test meals had a negative A.C.R.A. titre, these results are independent of the type of food used and are obviously due to whether the sample of gastric contents contained saliva or not. Two different interpretations of these results are possible:

(1) There is a relative absence of mucus in the gastric juice unless saliva is added to the food, which suggests that the healthy stomach does not ordinarily secrete mucus in the gastric juice, in response to normal food. (2) Larger amounts of mucus are present in the gastric juice when saliva is added to the food as compared to when it is not. This suggests that most of the mucus in gastric juice is of salivary origin.

In the case of the samples of fasting contents, the A.C.R.A. titres were invariably high (fifth to tenth tube). This can be explained because a lot of saliva is normally swallowed during sleep. However, it is impossible to be certain that the fasting juice has no gastric component of mucus.

TOTAL AMOUNT OF MUCUS A comparison of the A.C.R.A. titres between samples with saliva in different studies shows that the concentration of mucus was higher in study A than in study B and higher in study $C$ than in study $D$. Since the average volumes of gastric juice aspirated were larger in the series with saliva as compared with the series with no saliva $(P<0.01)$, the mean values being $87.0 \mathrm{ml} . \pm 8.21$ and $51.5 \mathrm{ml} . \pm 8.27$ in the samples with saliva and the samples with no saliva respectively in study A (Table III), this indicates that our results were not due to dilution, and not only the concentration but also the total amount of mucus in gastric aspirates was higher in the samples with 
TABLE III

MEAN VALUES FOR VOLUME OF GASTRIC CONTENTS RECOVERED FROM 13 INDIVIDUALS IN STUDY A (FLUID MEAL SIP BY SIP AND BY INTUBATION) AND FROM SEVEN INDIVIDUALS IN STUDY B (FLUID MEAL GULPED VERSUS INTUBATION)

\begin{tabular}{|c|c|c|c|}
\hline \multirow[t]{2}{*}{ Experiment } & \multicolumn{2}{|c|}{ Volume of Gastric Contents ( $\mathrm{ml}$. ) } & \multirow[t]{2}{*}{ Probability } \\
\hline & With Saliva & With No Saliva & \\
\hline $\begin{array}{l}\text { Study A } \\
\text { Mean } \\
\text { S.E. } \pm\end{array}$ & $\begin{array}{c}87.0 \\
8.21\end{array}$ & $\begin{array}{c}51 \cdot 5 \\
8.27\end{array}$ & $P<0.01$ \\
\hline $\begin{array}{l}\text { Study B } \\
\text { Mean } \\
\text { S.E. } \pm\end{array}$ & $\begin{array}{r}67 \cdot 57 \\
7 \cdot 17\end{array}$ & $\begin{array}{r}55 \cdot 57 \\
5 \cdot 37\end{array}$ & 0.3 (N.S.) \\
\hline
\end{tabular}

saliva than the samples with no saliva, and this closely correlates with the amount of saliva swallowed with food and with the concentration of mucus in the saliva, because in study A where fluid meals were taken sip by sip in small sips, the amount of saliva swallowed would naturally be larger as compared with study B where the meal was taken as a 'long drink' or in one 'gulp', a mode of ingestion which does not encourage much admixture of saliva with the meal. Similarly larger amounts of saliva of a higher mucus content are swallowed with masticatory meals (study C) as compared with 'sloppy' meals used in study $\mathrm{D}$, because the chewing movements of the jaw not only increase the total amounts of saliva secreted but also result in an increased concentration of mucus in saliva (Dewar, 1952; Dewar and Parfitt, 1954; Knox and Still, 1953; Malhotra et al., 1965). This can explain why there was a higher concentration of mucus in samples with saliva obtained after eating masticatory meals as compared with sloppy meals. This also is probably one of the main reasons why Glass and Boyd (1948) found $60 \%$ lower values of mucin in an in vitro mixture of equal parts of gastric juice and saliva than in the same uncontaminated gastric juice. Since they had used unstimulated saliva, which ordinarily is expected to contain very little mucus (Dewar, 1952; Malhotra, 1965), and since the gastric juice in their experiment had not been obtained after a stomach wash, it was rich in mucus just like our fasting samples, a situation was created in which the addition of saliva to the sample of gastric juice resulted in a dilution of the concentration of mucus originally present in the gastric juice. Their result is thus in consonance with our study B, but contrary to our studies $\mathrm{A}, \mathrm{C}$, and $\mathrm{D}$ in which the addition of saliva to the gastric juice in vivo markedly increased the concentration of mucin, because the mucus concentration greatly increases in stimulated saliva as compared with unstimulated saliva. Gastric mucus is, however, present in the gastric juice under abnormal conditions such as gastritis or duodenal reflux.

The absence of mucus from samples in studies in which the meals were introduced through the Ryle's tube thus 'excluding' saliva is extraordinary in view of the findings of others that the effects of mechanical irritation of the gastric mucosa as well as of eating are similar because these result in an increased outpouring of mucus from the epithelium (Wolf and Wolff, 1948). The explanation of our results probably lies in the fact that the concept of a layer of gastric mucus smeared over the mucus membrane does not give a true picture of the relation of gastric mucus to the gastric epithelium where the 'cell itself forms a part of the mucus layer', and, as pointed out by James (1957), the intracellular gastric mucus is adherent to the surface epithelium and continuous with plugs of mucus filling the foveolae, being functionally an additional layer of the stomach lining' and, therefore, it did not appear in our aspirates. The fact that in six of our subjects when $100 \mathrm{ml}$. of $5 \% \mathrm{NaHCO}_{3}$ was introduced the A.C.R.A. titres rose markedly even though the preceding runs of test meals had shown no mucus supports this possibility. This is also in agreement with the observations of several workers who have found that many particles of mucus adhere to the gastric mucous membrane and are not recovered through the gastric tube (Glass and Boyd, 1948; Wolf and Wolff, 1948).

In our subjects we had three individuals whose samples were heavily bile stained. In two of them, who showed a high A.C.R.A. titre (seventh to twelfth tube), the $p \mathrm{H}$ of the samples was between 7 and 7.4. In the case of the third, despite heavy bile staining, the $p \mathrm{H}$ was acidic and the A.C.R.A. titres were low (third to fourth tube). This would indicate that mere regurgitation of bile cannot dissolve mucus, nor does it affect the mucus concentration due to the mucus components of bile contaminating the gastric juice, but if there is a fall in acidity, the intracellular mucus is removed from the epithelial cells and can be aspirated through the tube. This is also supported by the observations of Ball and James (1961) and the results of our experiments in which we instilled in the stomach $100 \mathrm{ml}$. of $5 \% \mathrm{NaHCO}_{3}$.

We also investigated two patients suffering from chronic gastritis. Both of them showed higher A.R.C.A. titres in samples with no saliva than our normal subjects, although the titres were not as high as in samples with saliva. It is very difficult to interpret these results, but we suppose that, although in a healthy state the stomach may not secrete mucus, it does so in the presence of chronic inflammation and in this respect may resemble the respiratory mucosa which secretes large quantities of mucus in chronic bronchitis and hardly any in the healthy state. 
RELATIONSHIP OF THE SALIVARY MUCUS TO PEPTIC ULCERATION The findings reported here show unequivocally that as a result of eating food two kinds of mucus may be simultaneously present in the gastric lumen: (1) salivary mucus which may, on coming into contact with gastric acid, precipitate as a layer of mucus smeared over the surface of the mucosa and the food, and (2) intracellular gastric mucus which is an integral part of the epithelial lining but ordinarily cannot be recovered through the tube unless it has been removed from the cell due to a fall in acidity. Furthermore, the amount of the salivary mucus in the stomach varies a great deal and depends upon the pattern of diet and eating, being more when masticatory food is eaten as compared with sloppy meals not requiring much chewing.

Since mucus has a protective role against peptic ulceration, it is clear that a person who swallows larger quantities of mucus-rich saliva with his food will have a better protection because of the added protective barrier of the salivary mucus. Apart from this, it has been shown that human gastric as well as salivary mucus contain an acid-inhibitory factor which causes a true reduction in acid output, which is not due to an antacid or buffering action of the mucin (Johnston, 1965; Brunschwig, Rasmussen, Camp, and Moe, 1942; Menguy and Smith, 1959). Another possible mode of this acid-inhibitory action of the added salivary mucus might also be, as explained by James (1957), that mucus is precipitated by the gastric juice on the surface of the epithelium and in this state might be less permeable to secretogogues, which are thus prevented from reaching the receptors. The results of our $p H$ determinations, which are being reported elsewhere (Malhotra, 1967 a), support this hypothesis, because in studies A, $C$, and $D$, although the test meals employed for 'oral' and 'tube' feeding were identical for each study, the mean $\mathrm{pH}$ values and the mean values for $\mathrm{HCl}$ were lower in the samples with saliva as compared with the samples with no saliva, indicating that the addition of saliva had reduced gastric acidity, probably due to its mucus content, in addition to its well-known buffering capacity due to its bicarbonate content.

The pattern of diet and eating exercises a profound influence on both the acid as well as the mucus, because, in general a masticatory mode of eating provides saliva of a higher buffer capacity and a larger mucus content which is competent to neutralize gastric acid on the one hand and reinforce the mucosal defence against both acid and bile, on the other, as already discussed (Malhotra, 1965; Malhotra et al., 1965; Malhotra, 1966a and b, 1967a). A 'sloppy' diet, on the contrary, has both these defects.
SUMMARY

A new and simple method of quantitative assay of gastric mucus based on the original A.C.R.A. technique of Burnet has been adapted to measure gastric mucus.

Results of quantitative assays of mucin in samples of gastric juice obtained after test meals employing both fluid and solid meals revealed that free mucus, unless due to ingested saliva, is ordinarily absent from the stomach and even the entry of a meal into the stomach does not result in the outpouring of mucus from the stomach.

Free mucus in the gastric juice, which is either in solution or in suspension, is of salivary origin and is swallowed with food in varying quantities, depending upon the manner of eating.

Masticatory foods produced higher concentration of mucus in the gastric juice as compared with solid food which did not require much mastication.

There was a close correlation between the concentration of mucin in the gastric juice and the amount of saliva swallowed.

The possible protective role of the added salivary mucus, by augmenting the gastric mucus defence barrier, is discussed.

\section{REFERENCES}

Ball, P. A. J., and James, A. H. (1961). The histological background of gastric ulcer. Lancet, 1, 1365-1367.

Brooks, J. R., Erskine, J. M., Gephart, T., Swaim, O., and Moore, F. D. (1950). The chloride output rate of the human stomach in healthy subjects and ulcer patients: the effects of vagotomy and acetylcholine. Surg. Gynec. Obstet., 90, 155-170.

Brown, J. B., Wright, H. D., and Limbacher. H. P. (1938). Studies on the chemistry of mixed saliva. III. The composition of saliva produced at varying rates of secretion by the same person with special reference to calcium and phosphorus. J. dent. Res., 17, 191-196.

Brunschwig, A., Rasmussen, R. A., Camp, E. J., and Moe, R. (1942). Gastric secretory depressant in gastric juice. Surgery, 12, 887-891.

Burnet, F. M. (1948). The mucinase of V. cholerae. Aust. J. exp. Biol. med. Sci., 26, 71-80.

Dewar, M. R. (1952). An investigation of the physical, chemical and bacterial properties of saliva, with special reference to mucin content, and their colrelation with susceptibility to dental caries. Ph.D. Thesis, University of London. Quoted by D. Afonsky (1961). Saliva and Its Relation to Oral Health. Univ. Alabama Press.

_- and Parfitt, G. J. (1954). Mucin content, physical properties of saliva and caries activity. J. dent. Res., 33, 751-756.

Glass, G. B. J., and Boyd, L. J. (1948). Studies on dissolved mucin (mucoprotein) of the gastric juice. I. Preliminary tests and the rationale for a new colorimetric quantitative method for the determination of dissolved gastric mucin. Rev. Gastroent., 15, 396-414.

Grant, R. (1945). Rate of replacement of the surface epithelial cells of the gastric mucosa. Anat. Rec., 91, 175-185.

-, Grossman, M. I., and Ivy, A. C. (1953). Histological changes in the gastric mucosa during digestion and their relationship to mucosal growth. Gastroenterology, 25, 218-231.

James, A. H. (1957). The Physiology of Gastric Digestion, pp. 128, 131. Arnold, London.

-, and Pickering G. W. (1949). The role of gastric acidity in the pathogenesis of peptic ulcer. Clin. Sci., 8, 181-210. 
Johnston, I. D. A. (1965). Reduction of gastric acid secretion without operation. In Recent Advances in Gastroenterology, edited by J. Badenoch and B. N. Brooke, pp. 110-111. Churchill, London.

Knox, K. W., and Still, J. L. (1953). Observations on salivary mucoids. J. dent. Res., 32, 379-385.

Malhotra, S. L. (1965). Peptic ulcer: role of saliva in its aetiology. J. Ass. Phycns India, 13, $907-914$

- (1966a). Genesis of peptic ulcer (Abst.). J. Ass. Phycns India, $14,96$.

(1966b). Pathogenesis of peptic ulcer. Proc. 3rd Wld Congr. Gastroent., Tokyo vol. II, 70-72.

- (1967a). Protective action of saliva in peptic ulceration. A study of its effect on gastric secretion by dye-dilution technique. Scand. J. Gastroent., 2, 95.
- (1967b). Effect of saliva in gastric emptying. Amer. J. Physiol., 213, 169-173.

-, Saigal, O. N., and Mody, G. D. (1965). Role of saliva in the aetiology of peptic ulcer. Brit. med.J., 1, 1220-1222.

Menguy, R., and Smith, W. O. (1959). Inhibition of gastric secretion in the rat by normal human gastric juice. Proc. Soc, exp. Biol. (N.Y.), 102, 665-667.

Palmer, E. D. (1952). Histology of the normal gastric mucosa: an investigation into the state of normalcy of the stomachs of persons without upper gastrointestinal complaints. Gastroenterology, 21, 12-23.

Wolf, S., and Wolff, H. G. (1948). Studies on mucus in the human stomach, estimation of its protective action against corrosive chemicals applied to the gastric mucosa and attempts at quantitation of gastric mucin by two chemical methods. Gastroenterology, 10, 251-255. 